\title{
Vorticity in the turbulent flow above variously rough surfaces
}

\author{
Radka Kellnerováa ${ }^{1, *}$, Klára Jurčáková ${ }^{1, * *}$, Pavel Procházka ${ }^{1, * * *}$, and Václav Uruba ${ }^{1, * * * *}$ \\ ${ }^{1}$ Institute of Thermomechanics, CAS, Prague, Czech Republic
}

\begin{abstract}
Highly turbulent flows above variously rough surfaces were investigated by means of Time-Resolved Particle Image Velocimetry in a wind tunnel. Proper Orthogonal Decomposition was applied to both velocity and vorticity data in order to detect dominant features in the flow based on turbulent kinetic energy and enstrophy, respectively. While both the shape and location of the POD patterns exhibited similarity with other studies, a systematic inconsistency in terms of contribution from the features to the enstrophy between the previously published papers and our results were found.
\end{abstract}

\section{Introduction}

The Proper Orthogonal Decomposition (POD) is nowadays a routine mathematical tool for an extraction of the dominant structures from the turbulent flow. The POD works best in the flows where chaotic turbulence can not be diagnosed by straight-forward methods, usually applied to compute a conditional average, or to provide an intermittency threshold test capable to detect low and high velocity regions. Especially the wall-bounded flows is very complex and typically contain vortical features of many scales and number of non-rotational motions in a circumfluent flow.

In such complex flows, POD is still capable to extract merit-based structures from the redundant noise of the turbulence. The term merit-based herein means that a physical property (e.g., velocity, vorticity, concentration) inside the structure is correlated with itself in both time and space. It is necessary to note that its correct physical meaning has to be carefully verified by a systematic comparison of the identified POD patterns with their counterparts in the real flow.

One of the widely recognised transient features in the fully turbulent flow are hairpins [1,2]. Basically, they consist of a laterally stretched head with a lateral component of rotation, and two longitudinally prolonged legs with longitudinal sense of rotation. Between the legs, the lowmomentum region is formed, while the high-momentum region occurs above the head front. The ideal shape of the hairpins may be, however, severely disturbed and broken in the highly turbulent flows. [3] reported messy dearranged structures reminding the "limbs" of the hairpins over the field of cubes.

The hairpins are known to form a packet, imprinting the tilted line-like region of high vorticity into to two-

\footnotetext{
*e-mail: radka.kellnerova@it.cas.cz

**e-mail: klara.jurcakova@it.cas.cz

***e-mail: prochap@it.cas.cz

****e-mail: uruba@it.cas.cz
}

dimensional plane. These horizontally aligned regions may be detectable by means of POD.

Several researches documented a strong connection between the most dominant POD modes based on the velocity data and those based on the vorticity (e.g., [5, 6]). According to these studies, the vorticity of the first few POD velocity modes agreed with the dominant modes of the vorticity. Moreover, the relative contribution to the enstrophy from the first POD vorticity mode achieves a significantly high value (up to $36 \%$ ) $[5,6]$. A similar percentage value of the relative contribution from the first mode can be observed with the POD applied to velocity data within the variously turbulent flows $[7,8]$. For instance, the first mode in the turbulent flow generated over a flat surface, hence according to [11] classified as slightly rough, reaches $20 \%$ of turbulent kinetic energy (TKE), while in the flow over a very rough surface the first mode attains $40 \%$.

The relative contribution of POD applied to velocity were therefore in agreement with the published values about POD of vorticity. In our study, the vorticity results, however, differed in one order of magnitude from the published ones. Hence, we revised the POD applied to vorticity within the different categories of flow in this paper.

\section{Experimental set-up}

The experiment was performed in a pressure-driven opencircuit wind tunnel with a test section of cross-sectional dimensions $250 \times 250 \mathrm{~mm}$ and a length of $3000 \mathrm{~mm}$ (see Fig. 1). Foremost, flat surfaces covered by roughness elements with the heights $0,1.6,5,8$ a $16 \mathrm{~mm}$ (labelled M1-M5) were studied as they produce a surface boundary layer flow (final models labelled SBLM1-SBLM5). Afterwards, the turbulence generators in the shape of spires were added in front of the corresponding surface models in order to generate an atmospheric boundary layer (labelled ABLM1-ABLM5). Finally, the spires were moved 


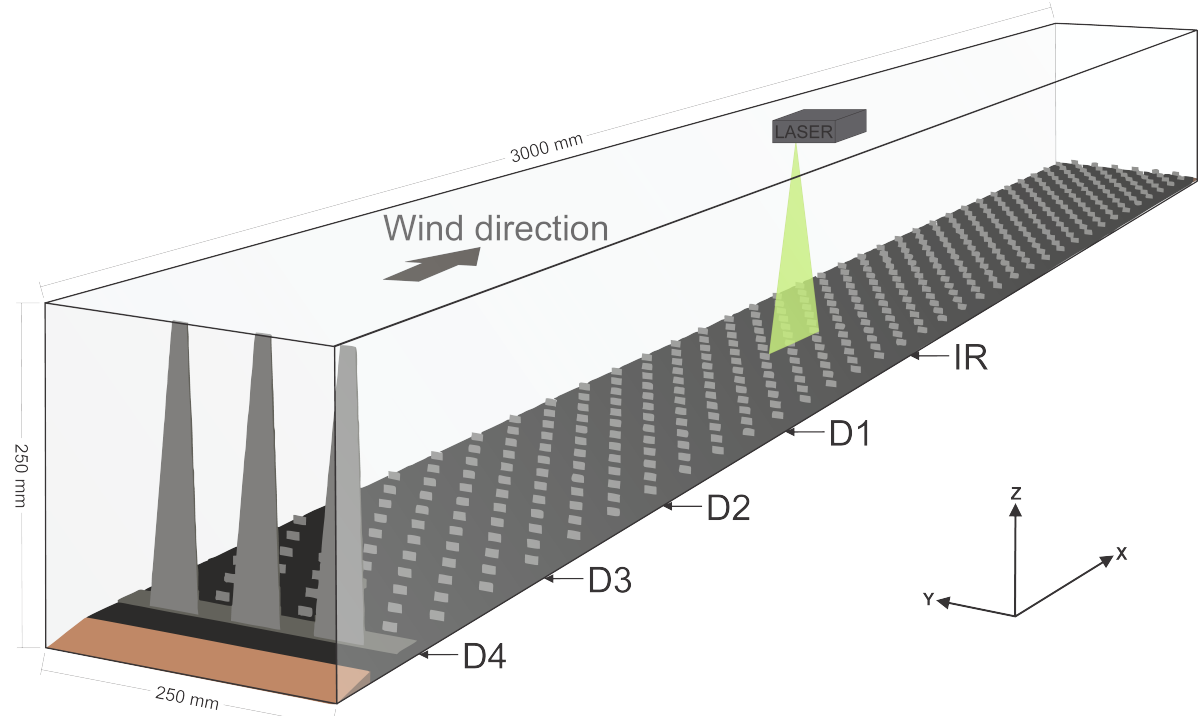

Fig. 1. Scheme of the wind tunnel test section with the spires installed at the beginning and with the surface covered by the roughness elements (ABLM4D4 case). The green triangle represents the laser sheet including the investigated region (IR). Labels D1-D4 denote the spires positions.

towards the investigated region (IR) by $500 \mathrm{~mm}$ in each step, so the distance $\mathrm{D}$ between the spires and IR was variable (labelled ABLMxD1-ABLMxD4).

The sampling frequency of the TR-PIV system was $100 \mathrm{~Hz}$, the number of acquired snapshots was 4000 and the corresponding acquisition time covered $40 \mathrm{~s}$. The IR was located at the tunnel centerline, $1824 \mathrm{~mm}$ downstream from the tunnel mouth. The studied 2-D plane had dimensions $90 \times 140 \mathrm{~mm}$, containing approximately $4000 \mathrm{vec}-$ tors, each of them represented a spatial average over the interrogation area $(32 \times 32 \mathrm{px} \approx 3.5 \times 3.5 \mathrm{~mm})$. With overlapping of $50 \%$, the final spatial resolution of PIV system reached $1.75 \times 1.75 \mathrm{~mm}$. The main nominal velocity, $U_{\text {red }}$, for the majority of the experiments was set to $5 \mathrm{~ms}^{-1}$ on the mouth of the wind tunnel. We tested also higher wind speeds, up to $13 \mathrm{~ms}^{-1}$, to check the influence of stronger momentum on the vorticity behaviour. The longitudinal (streamwise) direction is denoted $\mathrm{X}$, the lateral one $\mathrm{Y}$ and the vertical one $\mathrm{Z}$, respectively.

\section{Results}

\section{Statistics}

The basic statistics including the mean longitudinal velocity, $U$, standard deviation $\sigma_{U}$ and $\sigma_{W}$, and mean momentum flux, $u^{\prime} w^{\prime}$ were published by [10]. The statistics normalised by the nominal velocity, $U_{\text {red }}$, for the SBLM1SBLM5 cases are depicted in Fig. 2 as an example. A step change visible in all the profiles around the normalised height $0.2 \delta$ is a consequence of the presence of the roughness elements.

\section{Proper Orthogonal Decomposition}

The POD groups motions, which exhibit signs of a correlation, into corresponding merit-based ensembles, and by this it offers an analysis of the coherent dynamics [4]. Briefly stated, POD collects the transient features together by finding their most appropriate representation, called basal vectors. While working with velocity, this representation expresses the highest TKE content in each vector from a statistical point of view. If working with vorticity, POD reveals enstrophy content in the flow. It is worthy to emphasize that due to a PIV system limitation, TKE consists of two velocity components (longitudinal and vertical) and enstrophy of one vorticity component (lateral) in this paper.

We performed POD with vorticity data and carried out geometrical patterns of four most dominant modes. We also evaluated the relative contributions from these particular modes with respect to the total enstrophy within the flow. The investigated region was identical for all the surface cases, with an exception brought by the necessity to cut off an area between the roughness elements since an enormous vorticity production takes place here. This vorticity enhancement caused by a presence of the elements can significantly influenced the POD results and blurred the vorticity in the remaining flow.

The results for the SBL cases confirmed that the geometrical pictures of the POD vorticity modes are very similar to those published in other studies (see Fig. 3). These geometries presumably represent a two-dimensional imprint from a packet of multiple hairpin structures, as was numerically modelled and experimentally documented by [2] and [1], respectively. Further, Fig. 3 indicates that the vertical dimension of the patterns as well as their elevation is increasing with the roughness elements height. The tilt angle of the high vorticity area with respect to the horizontal surface is decreasing with the elements height, attaining $10^{\circ}$ for the smooth SBLM1 case to $7^{\circ}$ in the rough SBLM5 case. This is in agreement with other published authors $[1,5]$. 

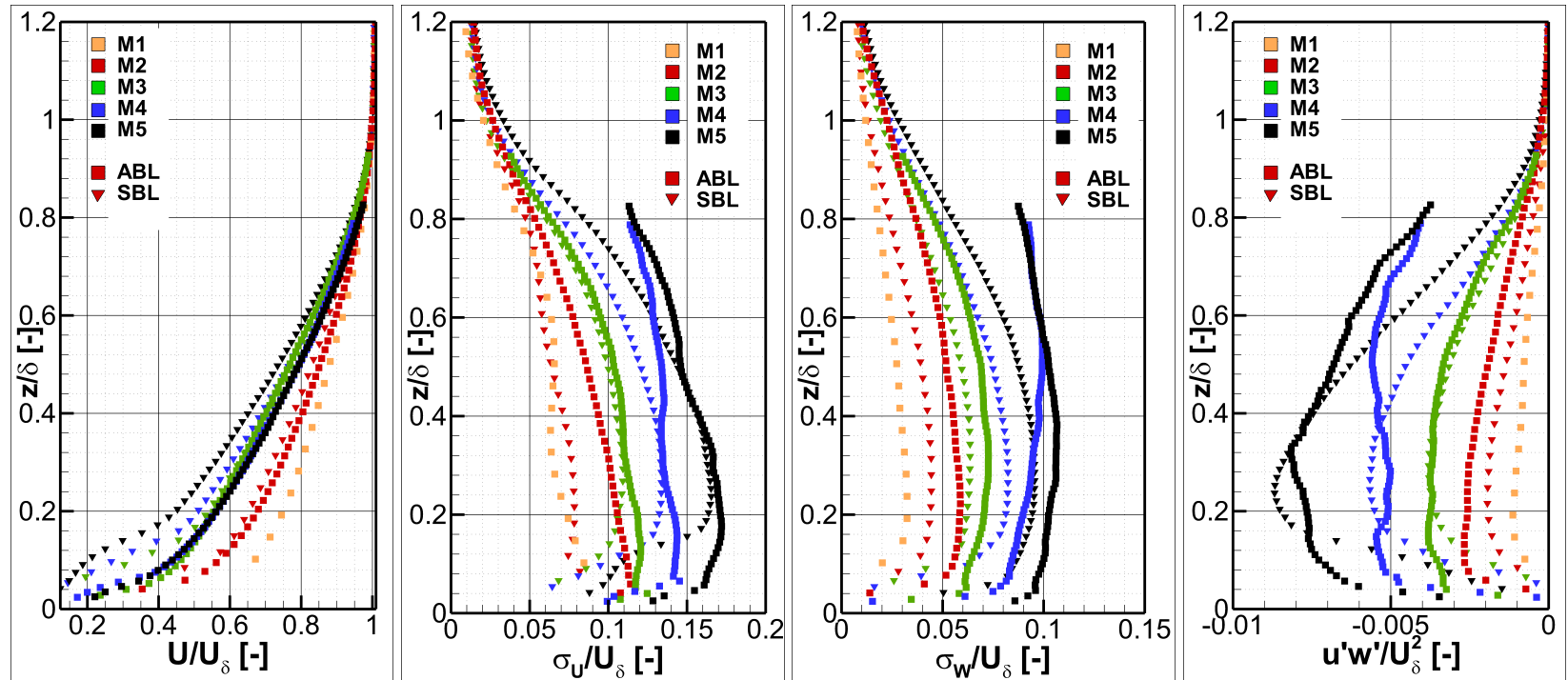

Fig. 2. Mean longitudinal velocity, longitudinal and vertical standard deviation and corresponding momentum flux. Data are taken from the SBLM1-SBLM5 (ABLM1=SBLM1), with a reference spire distance D4. Each profile is an average along the horizontal coordinate within the investigated region.
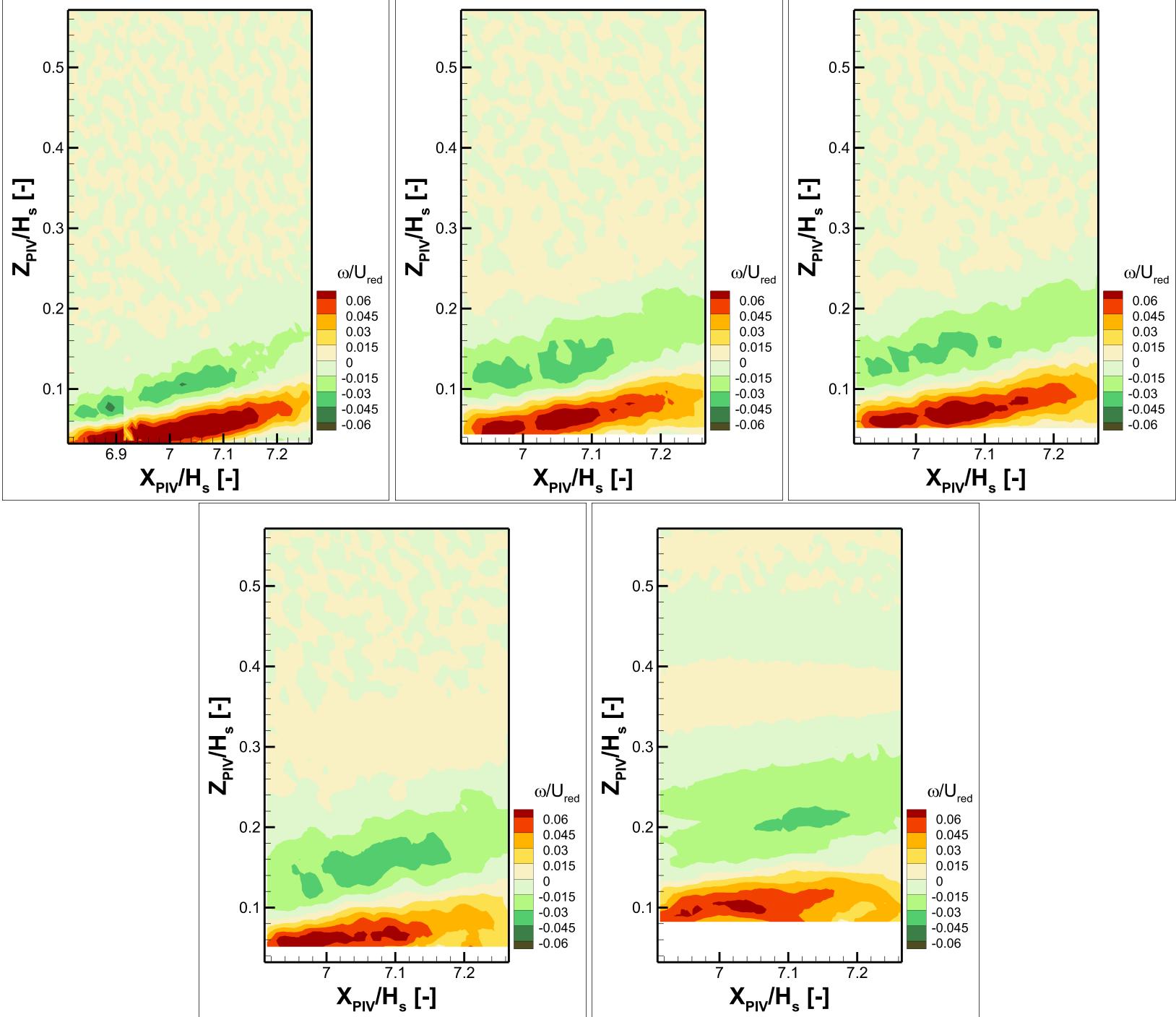

Fig. 3. The first POD modes of vorticity for the surface boundary layer SBLM1-SBLM5 with a reference spire distance D4, from left upper corner to right bottom corner. $\mathrm{H}_{S}$ is the inner height of the test section. Flow comes from left to right. 


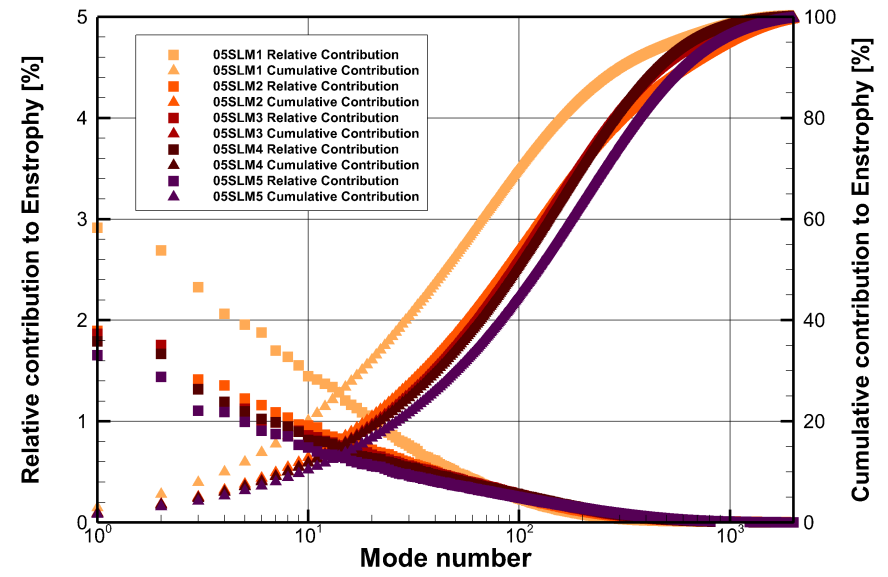

Fig. 4. The relative contribution of the POD modes to the total vorticity for the SBLM1-SBLM5 cases with a reference spire distance D4. The abscissa represents the mode number. Left coordinate represents the relative contribution, right coordinate represents the cumulative contribution.
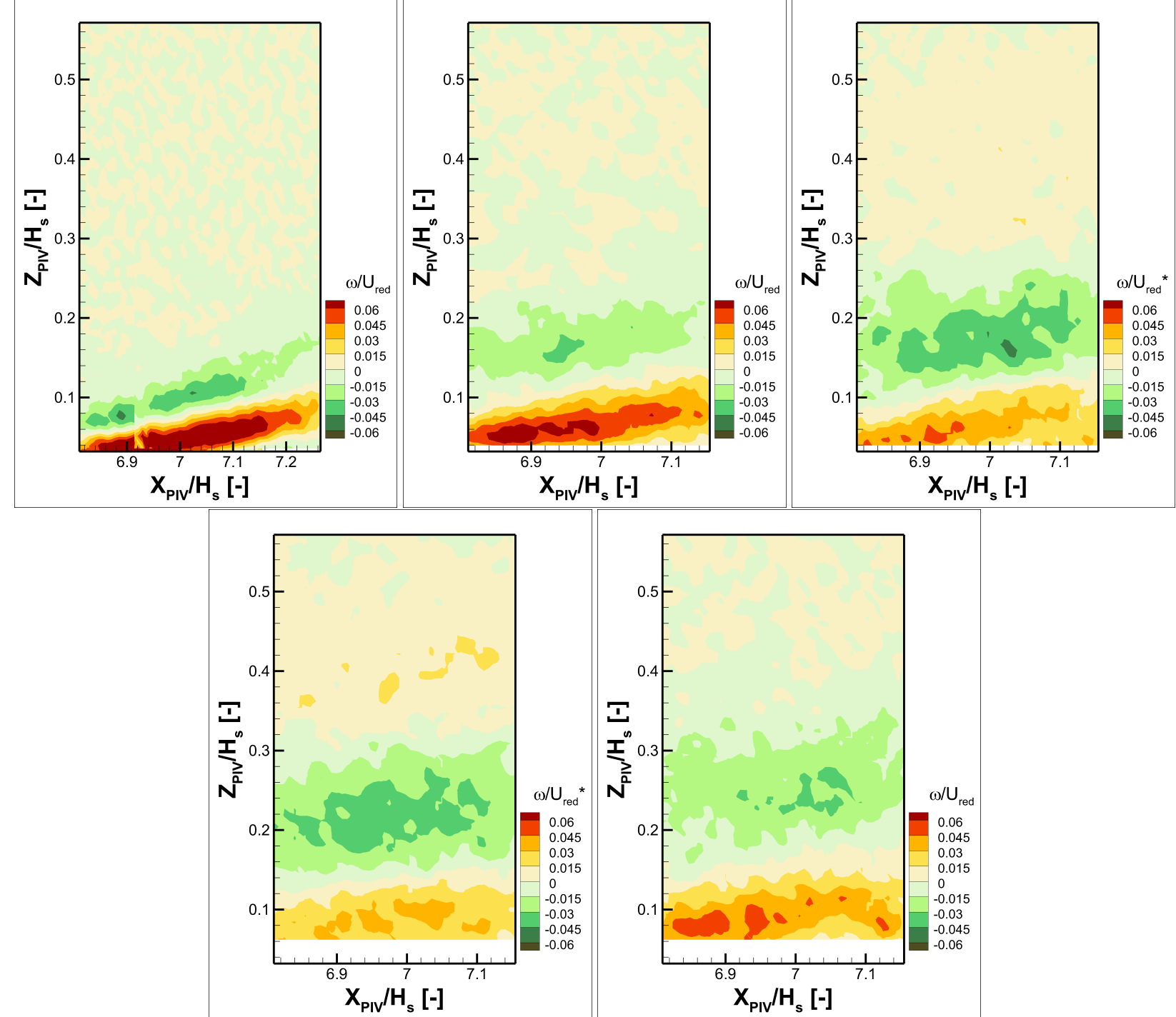

Fig. 5. The first POD modes of vorticity for the atmospheric boundary layer ABLM1-ABLM5 with a reference spire distance D4, from left upper corner to right bottom corner. $\mathrm{H}_{S}$ is the inner height of the test section. Flow comes from left to right. 


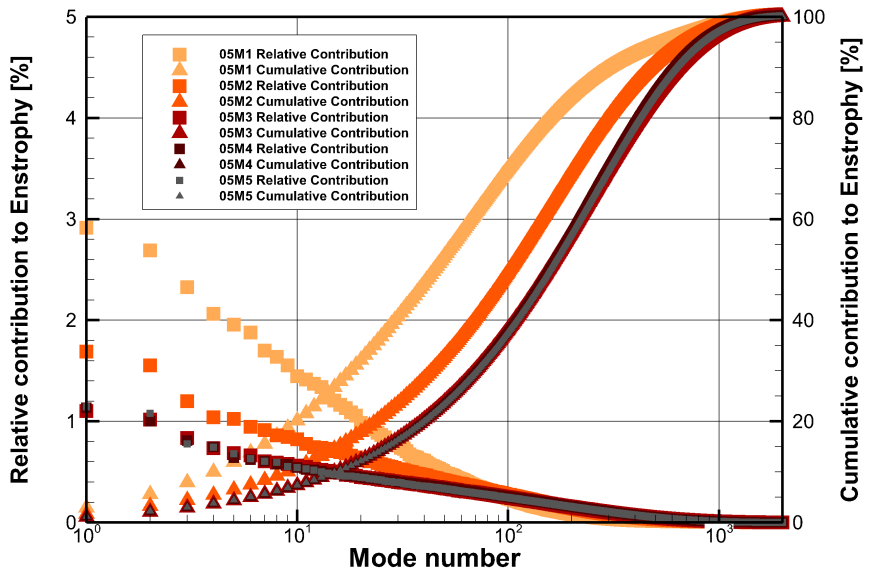

Fig. 6. The relative contribution of the POD modes to the total vorticity for the ABLM1-ABLM5 cases with a reference spire distance D4. The abscissa represents the mode number. Left coordinate represents the relative contribution, right coordinate represents the cumulative contribution.
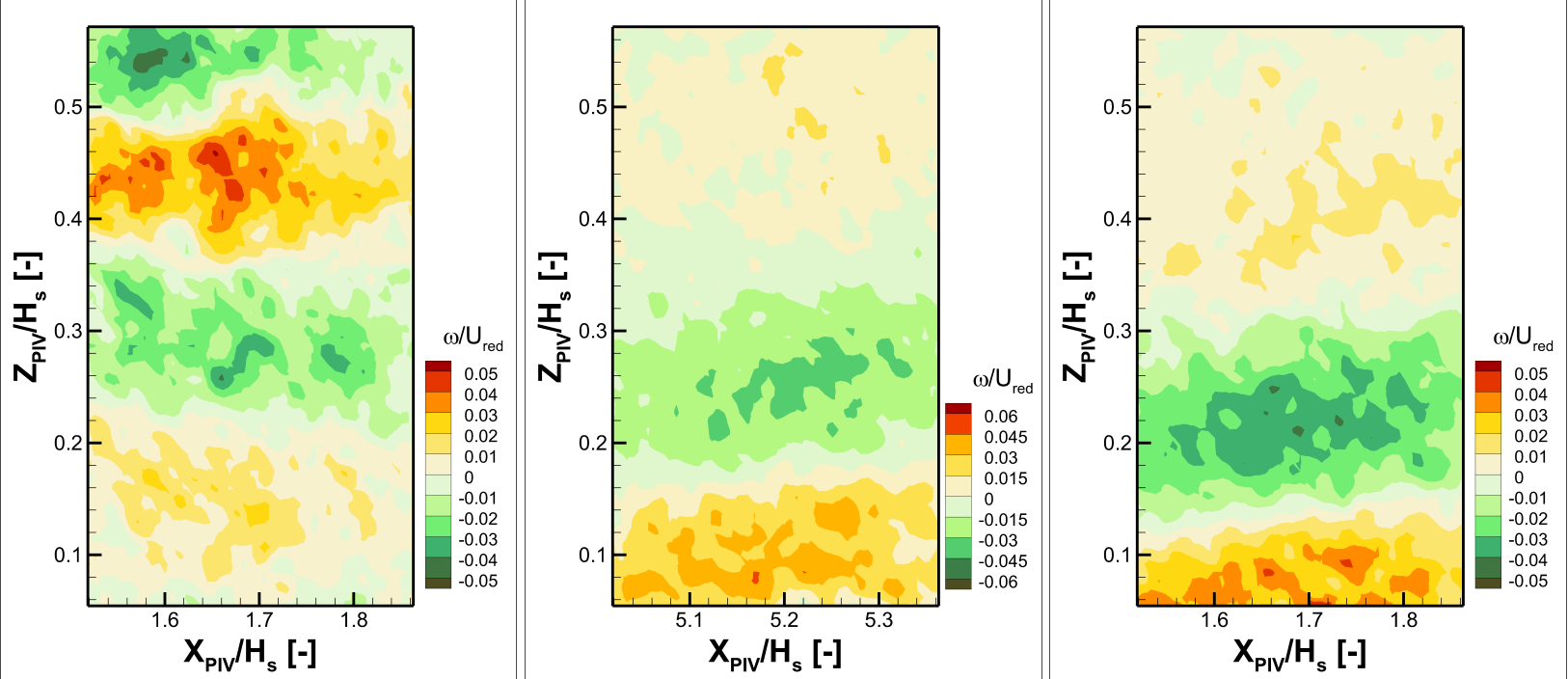

Fig. 7. The first POD modes of vorticity for the atmospheric boundary layer with variable distances of the spires ABLM4D1, ABLM4D3 and ABLM4D4, from left to right. $\mathrm{H}_{S}$ is the inner height of the test section. Flow comes from left to right.

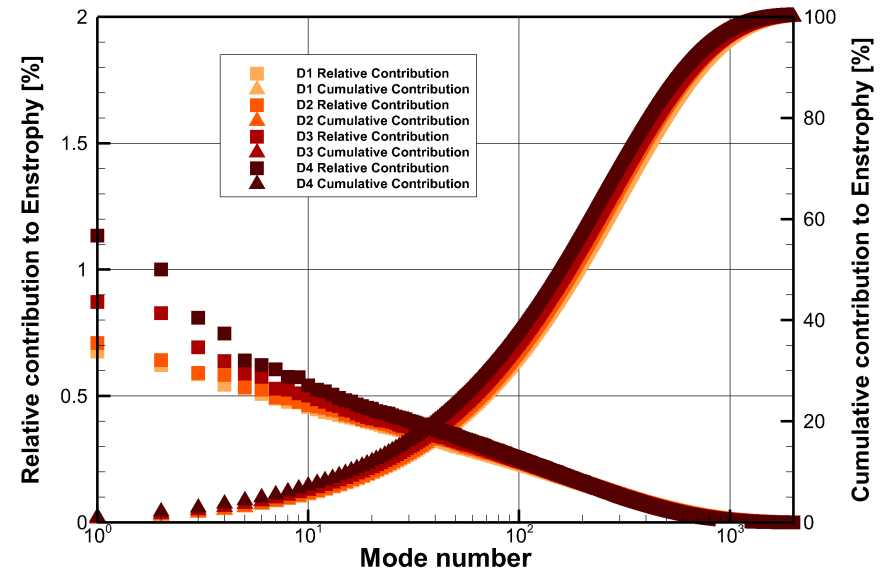

Fig. 8. The relative contribution of the POD modes to the total vorticity for the ABLM4D1-ABLM4D4 cases. The abscissa represents the mode number. Left coordinate represents the relative contribution, right coordinate represents the cumulative contribution. 
However, our analyses showed that the first few vorticity modes occupy very little percentage of the total enstrophy. Fig. 4 shows the relative contribution from the first mode being only $3 \%$, while the other surfaces exhibit even less contribution, approximately $2 \%$. Recalling our POD applied to velocity in [9], the strongest velocity mode captures $20 \%$ in the SBLM1 case and almost $40 \%$ in SBLM5 case. The rate of convergence of the relative contributions - labelled cumulative contribution herein - was very fast for the velocity data. The first hundred velocity modes covered unambiguous majority of the total TKE, from $80 \%$ (in SBLM1 case) to 90\% (in SBLM5). The rate of convergence is an important parameter since it demonstrates the degree of organisation in the flow.

On the other hand, the rate of convergence for the vorticity data is much slower. The first hundred vorticity modes contain $70 \%$ of the total enstrophy over the surface SBLM1, while the other SBL surfaces got even worse score with approximately $50 \%$ of enstrophy covered by the same number of modes.

The similar result was observed for all the surfaces of the atmospheric type (ABLM1-ABLM5), where the thickness and the momentum flux of the boundary layer were pronounced by the spires. Fig. 5 depicts the same first POD modes based on the vorticity data for ABLM1-ABLM5. The geometrical patterns are more vertically extensive and occupy the higher elevations. Their edge is also significantly ragged in a comparison to the modes from SBLM1-SBLM5 cases, where patterns have a smooth rim. The tilt angle decreases with the increasing roughness, attaining $10^{\circ}$ for ABLM1 case and $8^{\circ}$ for ABLM5 case.

As well as in the previous cases, the amount of enstrophy captured in the most dominant vorticity mode is remarkably low since the first mode does not exceed $3 \%$ and this amount further decreases with the increasing roughness (Fig. 6). While the enstrophy contribution drops with increasing turbulence intensity in the flow, the amount of turbulent kinetic energy inside the velocity modes increases, on the contrary.

The nominal wind speed in the range from $5 \mathrm{~ms}^{-1}$ to 13 $\mathrm{ms}^{-1}$ was found to have no effect neither on the geometry of the dominant modes nor on the tilt angle of the structure.

The same results were found also for the different distances D1-D4 between the investigated region and the spires. The example can be seen for the atmospheric boundary layer case ABLM4, labelled as ABLM4D1ABLM4D4, in Fig. 7. The patterns are very similar to each other at three distances D2-D4. Just behind the spires, at the distance D1, the geometrical appearance of the modes strikingly changes. As the spires completely disturb the flow and produce lot of vortical motions, the vorticity POD patterns lose their typical spatial distribution and become more homogenously located in the space. Notwithstanding, Fig. 8 proves the relative contribution of the POD modes to enstrophy just behind the spires to be even smaller, reaching value of $0.7 \%$ (compare to value of $1.3 \%$ at position $\mathrm{D} 4)$.

\section{Conclusion}

The systematic discrepancies in the relative contribution to the enstrophy in the flow were found and verified between our study and published studies of other authors. Although the spatial shape of the POD modes were extremely similar to the published findings and the tilt angle was found out to be identical, the contribution to the enstrophy was strikingly off. Since the mathematical method POD was used in the same manner, the data were collected by the similar PIV devices and the same physical properties were analysed as in other authors works, we could not provide any explanation to clarify such difference.

The goal in the near future is to test different versions of the Proper Orthogonal Decompositions as well as various de-noising methods applied to raw data to enlighten such a significant divergence between the results.

The authors kindly thank the Czech Science Foundation GA CR - GA15-18964S for its financial support.

\section{References}

[1] R. J. Adrian, C. D. Meinhart and C. D. Tomkins, Vortex Organization and Structure in the Outer Region of a Turbulent Boundary Layer, J. Fluid Mech., Vol. 240, pp. 1-55, (2000)

[2] J. Zhou, R. J. Adrian , S. Balachandar, T. Kendall, Mechanism for Generating Coherent Packets of Hairpin Vortices in Channel Flow, J. Fluid Mech., Vol. 387, pp. 353-396, (1999)

[3] O. Coceal, A. Dobre, T. G. Thomas, S. E. Belcher, Structure of Turbulent Flow Over Regular Arrays of Cubical Roughness, J. Fluid Mechanics, 589, pp. 375409, (2007)

[4] N. Aubry, R. Guyonnet, R. Lima, Spatiotemporal Analysis of Complex Signals: Theory and Applications. Journal of Statistical Physics, Vol. 64, pp. 683739, (1991)

[5] R. Gurka, A. Liberzon, G. Hetsroni, POD of vorticity fields: A method for spatial characterization of coherent s tructures, International Journal of Heat and Fluid Flow, Vol. 27, Issue 3, pp. 416-423, (2006)

[6] J. Kostas, J. Soria, M. S. Chong, A comparison between snapshot POD analysis of PIV velocity and vorticity data, Experiments in Fluids, Vol. 38, Issue 2, pp. 146-160, (2005)

[7] R. Kellnerová, L. Kukačka, K. Jurčáková, V. Uruba, Z. Jaňour, PIV measurement of turbulent flow within a street canyon: Detection of coherent motion. Journal of Wind Engineering and Industrial Aerodynamics Vol. 104-106, pp. 302-313, (2012)

[8] R. Kellnerová, V. Fuka, V. Uruba, K. Jurčáková, Š. Nosek, H. Chaloupecká, Z. Jaňour, On Street-Canyon Flow Dynamics: Advanced Validation of LES by Time-Resolved PIV. Atmosphere, Vol. 9(5), pp. 161, (2018)

[9] R. Kellnerová, K. Jurčáková, V. Uruba, Z. Jaňour, Non-rotational coherent structures in the flow with 
different degree of turbulence, American Meteorological Society, 22nd Symposium on Boundary Layers and Turbulence, Salt Lake City, Utah, USA, (2016)

[10] K. Jurčáková, R. Kellnerová, P. Procházka, P. Antoš, Spectral characteristics of turbulent boundary layers - comparison of Particle Image Velocimetry and Thermal Anemometry, Proceeding EFM 2018, (2018)

[11] VDI, Physical Modelling of Flow and Dispersion Processes in the Atmospheric Boundary Layer - Application of Wind Tunnels, (2000) 